\title{
EDITORIAL
}

\section{IN THE APRIL 2009 ISSUE OF CLINICS}

\author{
Mauricio Rocha-e-Silva, Editor
}

doi: $10.1590 / \mathrm{S} 1807-59322009000400001$

In this April issue of Clinics we highlight a study by Paval et al. who evaluated the anti-arthritic potential of the plant Justicia gendarussa using two different models, namely arthritis induced either by injecting Freund's complement adjuvant or bovine type II collagen into the plantar surface of the hind foot of rats. Paw edema was the measured parameter and authors conclude that the ethanolic extract of the plant exhibited significant anti-arthritic activity that was statistically similar to that of aspirin. We publish 13 reports on original clinical science, 2 in basic research and 3 case reports.

Cabrera et al. analyzed medications that act on the cytochrome p450 system (continuously used medications with hepatic metabolism via CYP450 classified as substrates, inducers or inhibitors) in 396 elderly individuals ( $\geq 60$ years old) and concluded that the elderly use high levels of medications that act on CYP450, thereby increasing the risk of drug interactions in a group that is already vulnerable to adverse drug effects.

Santos LM et al. evaluated the effects of intrathecal morphine on pulmonary function, analgesia, and morphine plasma concentrations after cardiac surgery in 42 patients randomized for general anesthesia or $400 \mu \mathrm{g}$ of intrathecal morphine followed by general anesthesia and concluded that morphine administration did not significantly alter pulmonary function; however, they claim that it improved patient analgesia and reduced morphine consumption and morphine plasma concentration.

Filiz et al. endeavored to determine whether persistently high postoperative serum carcinoembryonic antigen in 114 patients surgically treated for colorectal cancer is of importance in the assessment of prognosis. They found that abnormal preoperative antigen levels significantly correlated with the depth of tumor invasion, lymph node metastasis, staging, and recurrence, whereas abnormal postoperative

Hospital das Clínicas, Faculdade de Medicina da Universidade de São Paulo - São Paulo/SP, Brazil.

mrsilva36@hcnet.usp.br antigen levels significantly correlated to the depth of tumor invasion, staging, and postoperative relapse. They conclude that the survival of patients with high postoperative antigen levels due to unknown reasons is the worst and that it may be extended if they are exhaustively tested with sensitive diagnostic methods and treated at an early stage.

Carvalho et al. analyzed the prevalence of methicillinsusceptible $S$. aureus and methicillin-resistant $S$. aureus as well as its antimicrobial susceptibility profile in saliva samples of 340 health professionals at a large public education hospital and found that methicillin-susceptible $S$. aureus occurred in $43.5 \%$ (148/340), while resistant $S$. aureus was found in $4.1 \%$ (14/340) of the studied cases. They conclude that this is important for both the education of health professionals and for preventative measures. Standard and contactprecautions should be employed in professional practice.

Millán et al. evaluated the results of monocular surgery under peribulbar anesthesia for large-angle horizontal strabismus in 92 consecutive patients who underwent surgery for large-angle horizontal strabismus (angle of 40 prism diopters or greater), and found that all patients with preoperative deviations up to $60 \mathrm{PD}$, had residual deviations under $15 \mathrm{PD}$. Some patients with preoperative deviations of $65 \mathrm{PD}$ and all patients with deviations over 65 PD had residual deviations over $15 \mathrm{PD}$. These underwent a second procedure with successful outcomes. They conclude that their ROC curve analysis showed that the cutoff point for obtaining a successful surgical result was 62.5 PD. No patient presented with a major limitation in respect of ocular movement.

Kara-Junior et al. compared the intraoperative safety of two techniques of capsulorhexis for intumescent white cataracts: traditional one-stage continuous curvilinear capsulorhexis and two-stage continuous curvilinear capsulorhexis and conclude that two-stage continuous curvilinear capsulorhexis helps prevent unexpected radial tears of the initial capsulotomy from high intracapsular pressure, sudden radialization of the continuous curvilinear capsulorhexis and other intraoperative complications due to high intracapsular pressure, thus providing a safe cataract surgery in cases of 
white cataracts. These findings were supported by ultrasonography.

Yanık B et al. examined the influence of obesity and inhaled steroids on bone mineral density in 46 postmenopausal asthma patients undergoing treatment with inhaled corticosteroids, in comparison with 50 matched untreated healthy volunteers. They found no correlation between bone mineral density and duration of asthma, but a slight positive protective effect of high body mass index against osteoporosis in the asthma patients, but this effect was overcome by time and menopause status.

Basyches et al. analyzed concentric and eccentric strength and endurance in 11 patients with unilateral intermittent claudication and found that strength and endurance in the symptomatic leg were lower during concentric compared to eccentric action but suggest that studies are recommended to investigate the mechanisms underlying these responses and to analyze the effects of interventions to improve concentric strength and endurance on functional limitations in patients with intermittent claudication.

Zanati et al. examined cardiovascular risk factor profiles and 24-month mortality in 75 patients with symptomatic peripheral arterial disease and found that hypertension and smoking were the most frequent risk factors, followed by diabetes. Other important factors were undertreated dyslipidemia, high fasting glycine levels, and myocardial hypertrophy. Thirty-four patients had been treated with aspirin. Overall mortality over 24 months was $24 \%$ and was associated with age and lack of use of aspirin, because no deaths occurred among those using this drug. No association was found between cardiovascular death (11 cases) and the other risk factors.

Rocha et al. evaluated the impairment of endothelium-dependent and endothelium-independent coronary blood flow reserve after administration of intracoronary acetylcholine and adenosine, and its association with hypertensive cardiac disease. They divided 18 hypertensive patients with normal epicardial coronary arteries on angiography into two groups according to left ventricular fractional shortening (higher or lower that 0.25 ) and conclude that in hypertensive patients without risk factors and without cardiac failure, endothelium-dependent and endothelium-independent coronary blood flow reserve vasodilator administrations had similar effects in patients with either normal or decreased left ventricular systolic function.

Westphal et al. compared variations of plethysmographic wave amplitude ( $\Delta$ Ppleth) to determine the percent difference between inspiratory and expiratory pulse pressure $(\Delta \mathrm{Pp})$ cutoff values for volume responsiveness in a homogenous population of 43 postoperative cardiac surgery patients. They found that $\Delta \mathrm{Ppleth}$ is well correlated with $\Delta \mathrm{Pp}$ and constitutes a simple and non-invasive method for assessing fluid responsiveness in patients following cardiac surgery.

Oliveira et al. determined the publication rate of 313 orally-presented abstracts from the 2003 Urological Brazilian Meeting, as well as the factors determining this publication rate. They find that $39 \%$ percent of the abstracts were published after a median time of 14 months (range: 1 to 51 months). This is comparable to that of international congresses. The subsequent publication of presented abstracts and the selection of prospective studies with stronger evidence should be encouraged and may improve the scientific quality of the meeting.

Castro et al. addressed the risks of hypothermia and hypoglycemia during a 10-km open-water swimming competition in order to alert physicians to the potential dangers of this recently-introduced Olympic event. The highest ranked elite open-water swimmers in Brazil were included. They conclude that hypothermia may occur during open-water swimming events even in elite athletes competing in relatively warm water, and that core temperature must be a chief concern of any physician during an open-water swim event. They stress that capillary glycemia may have positive effects on performance and suggest further studies to include more athletes in a controlled setting.

Emre et al. compared the effects of intraperitoneal honey and sodium hyaluronate-carboxymethylcellulose (SEPRAFILM $^{\mathrm{TM}}$ ) for the prevention of postoperative intra-abdominal adhesions in rats, and suggest that both honey and Seprafilm ${ }^{\mathrm{tm}}$ decrease the incidence of adhesions in the rat cecal abrasion model. They also state that although the mechanism of action is not clear, intraperitoneal administration of honey was effective, and that the outcome of this study demonstrates that honey is as effective as Seprafilm ${ }^{\mathrm{tm}}$. 